\title{
Mutual information in classical spin models
}

\author{
Johannes Wilms ${ }^{1}$, Matthias Troyer ${ }^{2}$, Frank Verstraete ${ }^{1}$ \\ ${ }^{1}$ University of Vienna, Faculty of Physics, Boltzmanngasse 5, 1090 Wien, Austria \\ ${ }^{2}$ Theoretische Physik, ETH Zurich, 8093 Zurich, Switzerland
}

\begin{abstract}
The total many-body correlations present in finite temperature classical spin systems are studied using the concept of mutual information. As opposed to zero-temperature quantum phase transitions, the total correlations are not maximal at the phase transition, but reach a maximum in the high temperature paramagnetic phase. The Shannon and Renyi mutual information in both Ising and Potts models in 2 dimensions are calculated numerically by combining matrix product states algorithms and Monte Carlo sampling techniques.
\end{abstract}

PACS numbers: 03.67.-a, 05.10.Ln, 05.70.Fh, 65.40.gd, 75.10.Hk, 89.70.Cf

\section{INTRODUCTION}

Classical statistical mechanics models have been studied extensively for many decades. The reason even the simple classical 2-dimensional Ising model has managed to stay interesting for so long is due to the non-trivial correlations arising in it, especially around the phase transition, manifesting themselves in the divergence of thermodynamic quantities such as heat capacity or the correlation length at the phase transition. In this paper, we propose to study another measure for correlations, namely, the mutual information between two parts of a system. This quantity originates from information theory and has been increasingly applied to problems in strongly correlated quantum many-body systems [1-12 but it is equally well suited to the study of classical models [13], which is the subject of this paper.

The outline of this paper is as follows: In section II. we will define mutual information and explain why it is a measure for correlations in a system, and what properties we expect it to have. We will proceed to show how it can be calculated efficiently for classical spin systems, present some unexpected results on the location of the maximum of the mutual information (section III), and finally attempt to give a possible physical interpretation of these results.

\section{MUTUAL INFORMATION}

\section{A. Definition and motivation}

The (Shannon) mutual information $I$ between any two, classical or quantum, systems $A$ and $B$ that have possible states $a$ and $b$ occuring with joint probability $p_{a b}$ can be defined as

$$
I(A, B)=S_{A}+S_{B}-S_{A B}=\sum_{a, b} p_{a b} \log \frac{p_{a b}}{p_{a} p_{b}}
$$

where $p_{a}=\sum_{b} p_{a b}$ and $p_{b}=\sum_{a} p_{a b}$ are the marginal probabilities, obtained by summing over all the states of the respective other system. Note how we have to sum over all the states $(a, b)$ of the total system.
The mutual information can be understood as a sort of distance (more precisely: Kullback-Leibler divergence) between the actual probability distribution of the system and a product distribution, thereby measuring the difference to a system whose subsystems are not correlated.

The above definition of mutual information is based on the Shannon definition of entropy. Shannon entropies like $S_{A}=\sum p_{a} \log p_{a}$ can be seen as the limit $\kappa \rightarrow 1$ of a Renyi entropy $S_{A}^{(\kappa)}=\frac{1}{1-\kappa} \log \sum_{a} p_{a}^{\kappa}$. One good reason to study Renyi and Shannon entropies is that they have a well defined operational meaning [14]. Shannon entropy provides an asymptotic description of the properties of a system (probability distribution), in the sense that it describes the average number of bits needed to encode a state of the system, where the average is taken over all the states of the system, using their respective probabilities of occurence. This is identical to the average amount of randomness that can be extracted from the system.

The concept of mutual information quantifies the amount of information that we acquire about a part of the system A by looking at its complement B. In contrast to a quantity like the correlation length which can be defined using first-oder correlation functions, the concept of an "order" of a correlation does not apply to mutual information in any of its versions. Instead, it really measures correlation in an information-theoretic sense; it gives you the amount of information (say, in bits) that you gain about one part of the system by looking at the other one, thereby making use of correlations of all orders. If entropy quantifies the amount of uncertainty in a system, $S_{A}+S_{B}-S_{A B}$ can be seen as the extra certainty, or knowledge, in the total system as opposed to considering the systems separately - that is the information that connects the systems, the mutual information.

The use of mutual information as a measure of correlation can also be understood by seeing it as the natural generalization of the entanglement entropy, an entanglement measure for pure quantum states, to finite temperature, i.e. mixed (Gibbs-Boltzmann) states: For pure states, the entropy of a subsystem describes the amount of information one part of the system has about the other, $S_{A}=S_{B}$. For mixed states however, the entropy of the total system has to be subtracted, and it is also possible 


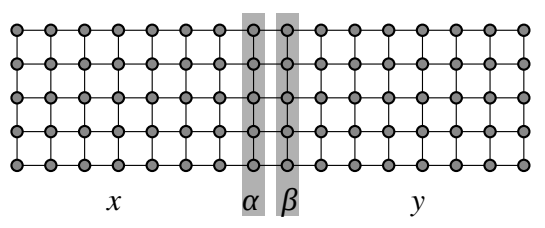

FIG. 1. Two subsystems and their interiors and boundaries

that $S_{A} \neq S_{B}$.

To conclude this section, let us explain why we think it is relevant to study the mutual information in classical spin models. First of all, our intuition tells us that the total amount of correlations in a Gibbs state should be maximized at the phase transition, as the correlation length is diverging exactly at that point. However, as we will point out, this notion is misguided: the mutual information is a measure not just of the 2-body but of all correlations present in the system, and this mutual information reaches a maximum away from criticality.

A second motivation for studying mutual information in classical spin systems is the fact that this provides a natural starting point for studying entanglement and correlations in quantum many body systems at finite temperature; almost no studies have been done in that respect, with the exception of the fact that a strict area law was proven at any finite $\mathrm{T}[13$. Contrasting classical to quantum behaviour is one of the main challenges in the field of quantum information theory, and this work provides a different approach to contrasting classical to quantum correlations.

\section{B. (Partial) partition functions}

We will now show how efficient access to "partial partition functions" can help us to reformulate the formula for mutual information in such a way that it no longer is a sum over all the states in the system - instead, we will just have to sum over the states of the "border" between the two systems [13. Of course, the sum over all states will simply be hidden in the partition functions.

We can then divide the description of a state of the system in two parts: the state of its "interior", and the state of its "border" with the other system. Here, the border is defined as the set of all those sites that share a bond with a site that belongs to the other subsystem. The rest of the sites makes up the interior. Figure1 1 illustrates this for a simple geometry, a strip that is divided in the middle, into "left" and "right" subsystems. Notice that in this geometry both $A$ and $B$ have three sides each of open boundaries which do not count as borders in our sense.

Let us now denote the separation into border and interior as $a=(\alpha, x)$ where $\alpha$ is the border and $x$ is the interior, and in the same way $b=(\beta, y)$.

Then the joint probability $p_{a b}$ factors into $p_{a b}=$ $p_{\alpha x \beta y}=p_{x} p_{\alpha x} p_{\alpha} p_{\alpha \beta} p_{\beta} p_{\beta y} p_{y}$ where $p_{x}$ describes the contribution from bonds between spins all in the interior of $A, p_{\alpha x}$ the contribution from the bonds between the interior and the border of $A, p_{\alpha}$ the one from bonds within the border, and so on. Plugging in this "factorization" of the probabilities, we find that we can rewrite the Shannon mutual information as

$$
\begin{aligned}
I(A, B) & =\sum_{\alpha x \beta y} p_{\alpha x \beta y} \log \frac{p_{\alpha x \beta y}}{\sum_{\beta^{\prime} y^{\prime}} p_{\alpha x \beta^{\prime} y^{\prime}} \sum_{\alpha^{\prime} x^{\prime}} p_{\alpha^{\prime} x^{\prime} \beta y}} \\
& =\sum_{\alpha \beta} p_{\alpha} p_{\alpha \beta} p_{\beta} \underbrace{\sum_{x} p_{x} p_{\alpha x}}_{Z_{A}(\alpha)} \underbrace{\sum_{y} p_{y} p_{\beta y}}_{Z_{B}(\beta)} \\
& \cdot \log \underbrace{\sum_{\sum_{\alpha \beta} p_{\alpha \beta^{\prime}} p_{\beta^{\prime}} p_{\beta^{\prime} y^{\prime}} p_{y^{\prime}}}^{\sum_{\beta^{\prime} x^{\prime}} p_{\alpha^{\prime} \beta} p_{\alpha^{\prime}} p_{\alpha^{\prime} x^{\prime}} p_{x^{\prime}}}}_{Z_{\tilde{B}}(\alpha)} .
\end{aligned}
$$

Some explanation is required: we have identified "partial" partition functions, for example $Z_{A}(\alpha)=\sum_{x} p_{x} p_{\alpha x}$ meaning the partition function of the system $A$ with the border $\alpha$ fixed - we are summing over all sites $x$ in the interior of $A$, but the border $\alpha$ of $A$ has some fixed value.

We have also defined partition functions of "extended" systems. For example, $Z_{\tilde{A}}(\beta)$ means the partition function of the enlarged system $\tilde{A}$, which has all of $A$ (both $x$ and $\alpha$ ) in its interior, and is bounded by $\beta$, which is the fixed border configuration for this partition function.

Therefore, if we have an efficient way to calculate such partition functions (crucially: with given fixed boundary conditions), we have reduced the sum over all the states to a sum over just the states of the borders.

The reduction of the mutual information to a sum that runs just over the boundaries of the systems is analogous to the approach in 13. where it was shown that this localisation of the mutual information in the boundary implies that an area law has to be obeyed.

\section{Renyi mutual information}

A very similar simplification as in the Shannon case also works for Renyi mutual information $I_{A B}^{(\kappa)}=S_{A}^{(\kappa)}+$ $S_{B}^{(\kappa)}-S_{A B}^{(\kappa)}$. Let us introduce modified partition functions $Z_{A}^{(\kappa)}(\alpha)=\sum_{x} p_{x}^{\kappa} p_{\alpha x}^{\kappa}$ and correspondingly for the other ones. Then we calculate the Renyi mutual information as

$I_{A B}^{(\kappa)}=\frac{1}{1-\kappa} \log \frac{\sum_{\alpha} p_{\alpha}^{\kappa} Z_{A}^{(\kappa)}(\alpha) Z_{\tilde{B}}(\alpha)^{\kappa} \sum_{\beta} p_{\beta}^{\kappa} Z_{B}^{(\kappa)}(\beta) Z_{\tilde{A}}(\beta)^{\kappa}}{Z_{A B}^{(\kappa)}}$

where we again only have to sum over boundaries. However, using the Renyi mutual information does, in our models, not seem to give much additional insight; it appears to be a smooth function of $\kappa$ around the Shannon value $\kappa=1$. 


\section{Monte Carlo}

While we will see how the partial partition functions can in many cases indeed be evaluated efficiently, there still remains a sum over the states of the borders in (1). While these borders between $d$-dimensional systems are just $d$-1-dimensional, the number of states is still exponentially large in the size of the border. While there might be a chance to carry out the sum exactly for small system sizes, we generally want to examine what happens towards the thermodynamical limit. Therefore, we will employ Monte Carlo methods to sample the exponentially large sum.

\section{Strip/cylinder geometry}

The preceding section showed how the calculation of mutual information can be simplified considerably when partial partition functions can be evaluated efficiently. We will now show how this efficient evaluation is possible for classical models, in the special strip-like geometry of figure 1 1 In particular, let us consider a very long strip of some given height, with a square lattice. Each column can be considered as a transfer matrix, applied to vectors at the (very far removed) left or right ends of the strip. Then, for an essentially infinitely long strip, the left and right boundary conditions become irrelevant, and each application of the transfer matrix corresponds just to multiplying the eigenvector corresponding to the largest eigenvalue $\Lambda$ by this largest eigenvalue.

Let us choose the two parts $A$ and $B$ simply as the left and right half of the strip, as shown in figure 1 Let the strip consist of $N$ columns in total, i.e. $N / 2$ in each of the subsystems, with the understanding that $N \rightarrow \infty$ such that above approximation is justified. The partition functions are then $Z_{A}(\alpha)=\Lambda^{N / 2-1}\langle\Lambda \mid \alpha\rangle, Z_{B}(\beta)=\Lambda^{N / 2-1}\langle\Lambda \mid \beta\rangle, Z_{\tilde{A}}(\beta)=$ $\Lambda^{N / 2}\langle\Lambda \mid \beta\rangle$, and $Z_{\tilde{B}}(\alpha)=\Lambda^{N / 2}\langle\Lambda \mid \alpha\rangle$. The partition function of the whole system, which is needed for normalization purposes, is $Z_{A B}=\Lambda^{N}$. Let us further introduce the shorthand $L(\alpha, \beta)=\langle\Lambda \mid \alpha\rangle\langle\Lambda \mid \beta\rangle$.

Let $q$ be the unnormalized Boltzmann weights, $q_{a b}=$ $\exp \left(-\beta E_{a b}\right)$ where $E_{a b}$ is the energy of configuration $(a, b)$. Let us now work with these $q_{a b}$ rather than the normalized probabilities $p_{a b}=q_{a b} / \sum_{a b} q_{a b}=q_{a b} / Z_{A B}$. The $q_{a b}$ can be factored exactly like the $p_{a b}$ in the previous section, and we then get the following formula for the mutual information

$$
I(A, B)=\frac{1}{\Lambda^{2}} \sum_{\alpha \beta} q_{\alpha} q_{\alpha \beta} q_{\beta} L(\alpha, \beta) \log \frac{q_{\alpha \beta}}{L(\alpha, \beta)}
$$

which is independent of $N$, so that the $N \rightarrow \infty$ limit is unproblematic.

\section{Eigenvector calculation}

For this special geometry, the calculation of (partial) partition functions essentially reduces to calculating the largest eigenvalue of the transfer matrix, and the corresponding eigenvector. The transfer matrix has a very special form: it is a matrix product operator (MPO).

The eigenvalues of an MPO can be calculated efficiently by a variational algorithm [15 18, and in fact calculating the extremal eigenvalues is particularly simple. The algorithm produces the eigenvector in the form of a matrix product state (MPS), which is a much more compact description than the full exponentially large vector would be. There is also no problem if we decide to connect the top and bottom of the strip by periodical boundary conditions, thereby turning the strip into a cylinder.

Of course, in any case the MPS is only an approximation, with the quality of the approximation depending on the chosen virtual bond dimension of the MPS. But for the Ising transfer matrix it turns out that we can get a reasonably good approximation with very low bond dimension like $D=8$ (of course, to completely avoid any errors, the bond dimension would have to grow exponentially with the system size; but we find that larger bond dimensions do not significantly change our results any more, so the small bond dimensions seem to catch the essential features of our system).

There is still an exponentially large sum in (2) though, which we cannot get rid of in principle, but which can be simplified as shown in the following:

\section{Simplifications}

We note that (2) would be a lot simpler if the logarithmic term wasn't in there, because then we would just have

$$
\sum_{\alpha \beta}\langle\Lambda \mid \alpha\rangle q_{\alpha} q_{\alpha \beta} q_{\beta}\langle\beta \mid \Lambda\rangle=\langle\Lambda|T T| \Lambda\rangle
$$

with $T$ the Ising transfer operator, and because that can be written as an MPO, this expression would be easy to calculate.

Of course we cannot simply drop the logarithmic term; but we can separate the logarithmic term into three parts, $\log \left(q_{\alpha \beta} /(\langle\Lambda \mid \alpha\rangle\langle\Lambda \mid \beta\rangle)\right)=\log q_{\alpha \beta}-\log \langle\Lambda \mid \alpha\rangle-\log \langle\Lambda \mid \beta\rangle$.

Now, $q_{a b}$ is actually an exponential, of the sum over all the bonds between two columns: $q_{a b}=$ $\exp \left(-K \sum_{i} \alpha_{i} \beta_{i}\right)$, where the sum goes over the rows $i$ and $\alpha_{i} \in\{-1,+1\}$ and $\beta_{i} \in\{-1,+1\}$ are the components of the configurations $\alpha$ and $\beta$, respectively.

The logarithm of the exponential is of course just the exponent $-K \sum_{i} \alpha_{i} \beta_{i}$, so we have to calculate

$$
-K \sum_{\alpha \beta}\langle\Lambda \mid \alpha\rangle q_{\alpha} q_{\alpha \beta} q_{\beta}\langle\beta \mid \Lambda\rangle \sum_{i} \alpha_{i} \beta_{i} .
$$


We can now consider the terms separately for each $i$, and notice that they are all local and can therefore efficiently be calculated as a contraction with suitably modified MPOs.

What about the remaining parts, with $\log \langle\Lambda \mid \alpha\rangle$ and $\log \langle\Lambda \mid \beta\rangle$ ? Let us look only at the one with $\log \langle\Lambda \mid \alpha\rangle$; the one with $\log \langle\Lambda \mid \beta\rangle$ is exactly the same, due to symmetry. We have

$$
\begin{aligned}
& -K \sum_{\alpha \beta} q_{\alpha} q_{\alpha \beta} q_{\beta}\langle\Lambda \mid \alpha\rangle\langle\Lambda \mid \beta\rangle \log \langle\Lambda \mid \alpha\rangle \\
= & -K \sum_{\alpha} q_{\alpha} \underbrace{\sum_{\beta} q_{\alpha \beta} q_{\beta}\langle\Lambda \mid \beta\rangle}_{\Lambda\langle\Lambda \mid \alpha\rangle}\langle\Lambda \mid \alpha\rangle \log \langle\Lambda \mid \alpha\rangle \\
= & -K \Lambda \sum_{\alpha} q_{\alpha}\langle\Lambda \mid \alpha\rangle^{2} \log \langle\Lambda \mid \alpha\rangle \\
= & -\frac{1}{2} K \Lambda \sum_{\alpha} q_{\alpha}\langle\Lambda \mid \alpha\rangle^{2} \log \langle\Lambda \mid \alpha\rangle^{2} \\
= & -\frac{1}{2} K \Lambda \sum_{\alpha} q_{\alpha}\langle\Lambda \mid \alpha\rangle^{2} \log q_{\alpha}\langle\Lambda \mid \alpha\rangle^{2} \\
& +\frac{1}{2} K \Lambda \sum_{\alpha} q_{\alpha}\langle\Lambda \mid \alpha\rangle^{2} \log q_{\alpha}
\end{aligned}
$$

where we introduced a $q_{\alpha} / q_{\alpha}$ unit term in the logarithm and used it to separate into two parts, one that has the form of an entropy, $H=-\sum_{\alpha} \pi_{\alpha} \log \pi_{\alpha}$, and one additional term where we again have the logarithm of an exponential, which becomes a sum of local terms that can be handled easily.

So what remains is just the entropy term that describes the entropy $-\sum_{\alpha} \pi_{\alpha} \log \pi_{\alpha}$ of the marginal distribution $\pi_{\alpha}=q_{\alpha}\langle\Lambda \mid \alpha\rangle^{2}$, normalized by $\sum_{\alpha} \pi_{\alpha}=\Lambda$. If we have the eigenvector $|\Lambda\rangle$, we can calculate this entropy: for small numbers of rows we can do it by exact summing, and for large numbers of rows it can be approximated by Monte Carlo sampling. In particular, note that for sampling the coefficients $\langle\Lambda \mid \alpha\rangle$ it is sufficient to know the eigenvector as a MPS, without ever needing it in exponentially large full form. We can do the sampling particularly efficiently by using Monte Carlo updates that sweep back and forth along the MPS and store intermediate contraction results.

\section{Nested rectangular geometry}

The MPS formalism is very well suited for the stripor cylinder-like geometry described before, and it has the huge advantage that it can just as well be used for not exactly solvable models. However, there is also a different kind of geometry that is another very natural candidate to be examined for the behaviour of the mutual information, namely, a nested geometry, where one of the systems is contained entirely within the other (in fact, this was the first system we decided to study). This geometry is shown in the small left inset of figure 3. While the MPS approach is not in principle unsuitable for this geometry, we still opted to use different techniques instead, which also has the advantage of serving as an independent way of verification of results. Unlike the MPS method, these kind of techniques will not work for all classical models, but only for exactly solvable ones.

The Fisher-Kasteleyn-Temperley method

This method can be formulated without any understanding of the (partial) partition functions as tensor network contractions. As detailed in for example [19, 20, the Ising partition function can be expressed as a sum of weighted dimer coverings on an extended orientable lattice. Handling of the fixed boundary conditions is the only essential new ingredient in our use of the FKT method, and it may be worth discussing it shortly: Being able to fix the directions of arbitrary spins in the lattice would be very similar to being able to solve the model in the presence of magnetic fields, which is not possible using this method. However, we only want to fix spins on the boundary of a system. Our approach to do this is to simply connect all these spins with bonds of infinite strength (this can in fact be done without introducing actual infinities). That way, their relative orientation is fixed, and all that remains is a factor of 2 in the partition function describing how the whole boundary can be flipped.

This sum of weighted dimer coverings can then be calculated as the Pfaffian of a matrix, the dimension of this matrix scaling linearly in the number of sites in the system. The Pfaffian can be easily calculated as the square root of a determinant, therefore with polynomial complexity.

\section{RESULTS}

We examined the behaviour of the mutual information as a function of the system parameters in the classical 2D Ising model on a square lattice, defined by the Hamiltonian $H=-J \sum_{\langle i j\rangle} s_{i} s_{j}$ where the sum is over all nearest neighbours and the $s_{k}$ take the values \pm 1 . One of the reasons for choosing the classical Ising model was the fact that there is just the one parameter $K=J / k_{B} T$ describing the bond strength in units of $k_{B} T$. We are however simulating finite systems, so clearly the system size, or more exactly sizes, are another group of parameters.

Let us first consider a system of some given fixed size though. How should the mutual information behave as a function of temperature? Clearly, for high temperatures (small $K$ ) the mutual information should tend to zero looking at one subsystem will not reveal anything about the other one, as all the spins are oriented randomly.

What happens for low temperatures? Again, the situation is easy to understand in our choice of the Ising model: There is a degenerate ground state manifold, 
spanned by the two states with either all spins pointing up or all spins pointing down. Therefore, at zero temperature (large $K$ ) the mutual information should be exactly one bit: If we see that one system has its spins pointing up, then we know that the spins in the other system will also point up, rather than down, and this is exactly the answer to one question with a binary answer.

What will happen at intermediate temperatures? The Ising model undergoes a phase transition at $K \approx 0.4407$, from the paramagnetic to the ferromagnetic phase. At a phase transition, the correlation length diverges in the thermodynamic limit. The mutual information describes correlations between the two subsystems. It is therefore natural to assume that the mutual information should have a maximum at the critical point, in a similar fashion like the entanglement entropy of ground states of several quantum models has been found to have a maximum (that actually becomes a singularity) at the quantum critical point [21, 22. However, in our case the mutual information does not have a maximum at the critical temperature:

\section{A. Strip/cylinder geometry}

Figure 2 shows the mutual information as a function of temperature, for the cylinder-like geometry, i.e. a strip with periodic boundary conditions in the finite dimension, with either 32 or 64 sites in that direction. It was calculated as described in section II C, using Monte Carlo sampling. The error bars due to Monte Carlo sampling are indicated.

We can see the predicted low- and high-temperature behaviour. There also is a maximum, as expected. However, the maximum is not at the critical point. It wouldn't be unreasonable to first assume that this is due to considering a finite system as opposed to the thermodynamic limit. The first indication that this is not the case is given by also plotting the heat capacity (up to constant factors), which is just a suitable derivative of the total partition function. The maximum of the heat capacity matches very well the critical point, thereby suggesting that the system in question is already a reasonably good approximation to the thermodynamic limit.

Also, once the system size is chosen suffiently large, the location of the maximum changes only very slightly with system size. It then lies deeply within the paramagnetic phase, and increasing the system size only appears to move it deeper into it. This is shown the lower part of figure 2, where in the bottom left we just multiplied the data for 32 rows with a factor of two and got very good agreement in the paramagnetic (high-temperature) phase, so clearly the maximum is not affected much by doubling the system size. In the bottom right the data for 32 rows is multiplied by a factor of two as well, but we subtracted 1 to get agreement in the limit of very low temperatures. It turns out we again find very good agreement for the whole ferromagnetic phase, right up
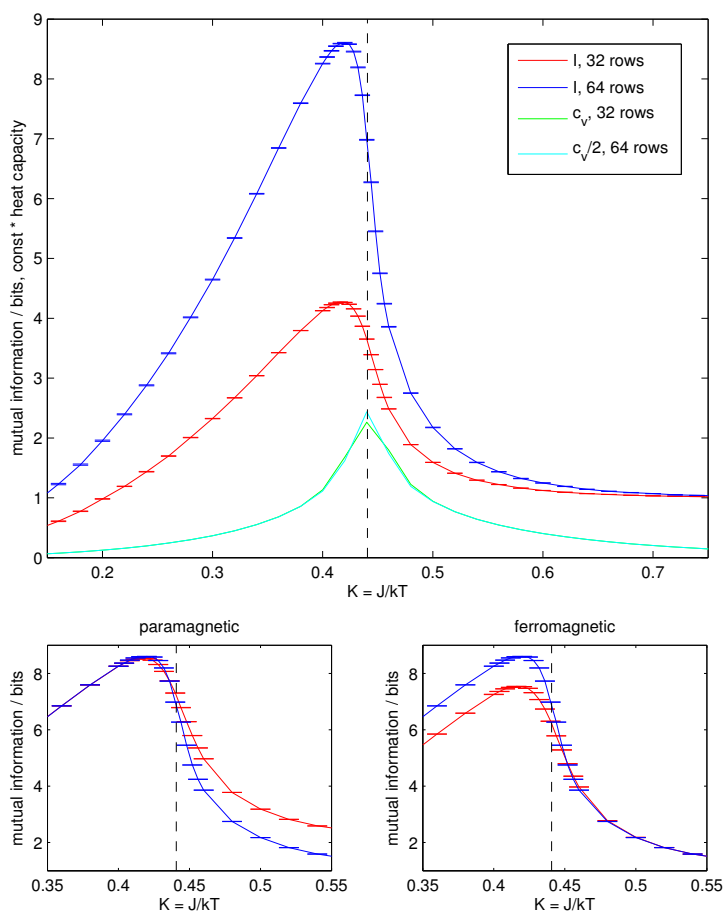

FIG. 2. Mutual information for the PBC strip geometry

to the critical point - not the maximum of the mutual information.

The bond dimension of the MPS approximation used for the data in the picture was just 8, which was chosen such that the dominant source of error is the Monte Carlo sampling, not the MPS approximation - choosing a smaller bond dimension and thereby allowing more samples to be taken in the same time appears the preferable approach. Only in the immediate vicinity of the phase transition with the diverging slope the error due to the MPS approximation is of the same order of magnitude as the sampling error (and the error bars should therefore no longer be taken too literally there) - however, this does not affect the location of the maximum in any way.

It should be noted that in order to reproduce our results for low temperatures, the eigenvector given by the MPS routines has to be suitably symmetrized. There, the ground state is almost degenerate, and instead of the actual ground state the MPS algorithms tend to find a different state within that approximate ground state manifold.

The fact that the curves for 32 and 64 rows agree so nicely does not mean that there is no influence of system size. For smaller system sizes, this is clearly the case. For these smaller system sizes, we can even work without Monte Carlo sampling, thus eliminating this as a possible source of error. We can also even use the exact eigenvector as opposed to its MPS approximation. We found 


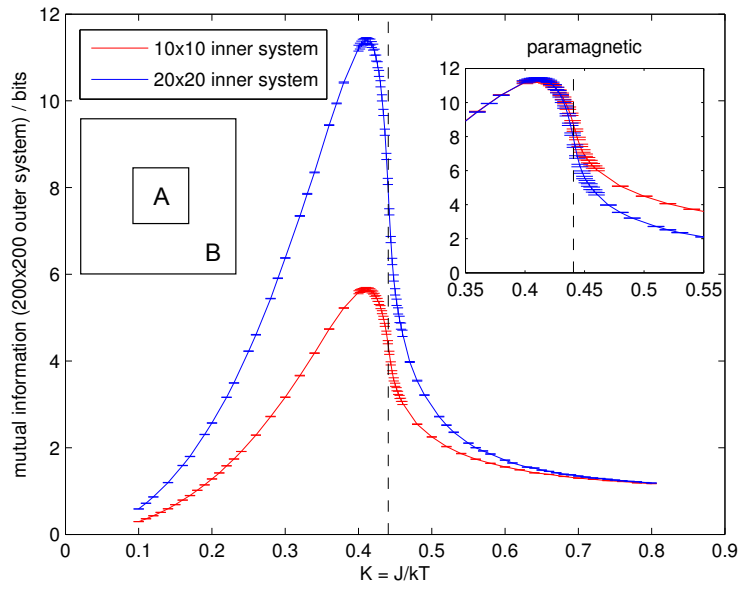

FIG. 3. Mutual information for the nested rectangular geometry

that in fact increasing the system size moves the maximum montonously deeper into the paramagnetic phase. The location of the maximum appears to move towards a value around $K \approx 0.41$, although it we could not fit any reasonable function and therefore cannot even see if there is convergence to any definite value at all. Quite as expected though, systems with periodic boundary conditions behave like much bigger systems with open boundary conditions.

It should be pointed out that the known location of the phase transition still appears to mark a relevant position in the plot: Just as has been found in 10 for different (quantum) models, it seems to be an inflection point; and in fact - at least in our case - one where the first derivative tends to minus infinity in the thermodynamic limit (as follows from the different scaling behaviours in the different phases).

\section{B. Nested rectangular geometry}

The results for the nested rectangular geometry are mainly presented to reinforce that the data shown in the previous section do not appear to be an anomaly, but describe the actual physics. Figure 3 presents data where we have a "square inside a square" geometry, with the outer square chosen so big as to hopefully avoid effects due to its size. This is confirmed by the fact that the data for an outer system with just $100 \times 100$ sites looks virtually identical, except for some slight finite-size effects around criticality.

Again, the maximum of the mutual information is clearly located in the paramagnetic phase, and in that phase it is again possible to "collapse" the data by multiplying the mutual information of the smaller system with a factor of two. The ferromagnetic phase appears to be somewhat more complicated in this geometry.

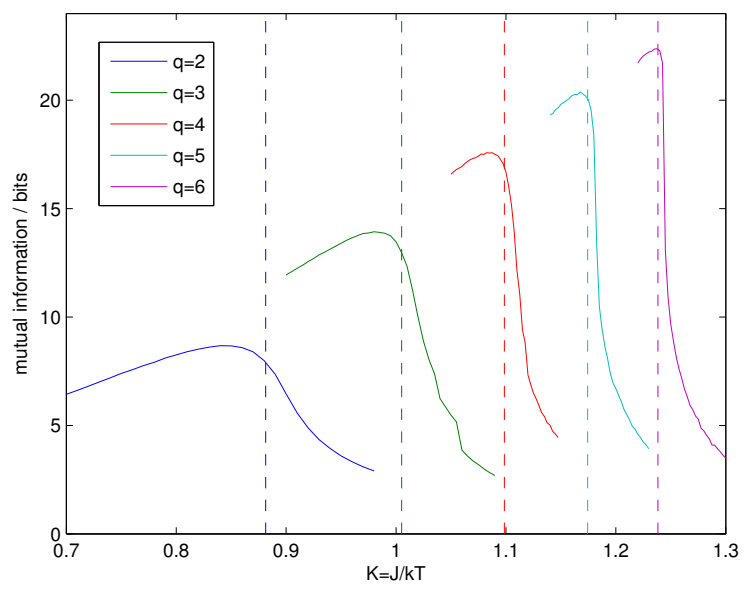

FIG. 4. Potts models with different values of $q$. Strip geometry (with open boundary conditions), 64 rows, MPS bond dimension $D=16$

\section{OTHER MODELS}

Let us take a brief look at a few other models: Figure 4 shows plots of the mutual information in Potts models, which are defined by the Hamiltonian $H=$ $-J \sum_{\langle i j\rangle} \delta\left(s_{i}, s_{j}\right)$, where the $s_{k}$ can now however take $q$ different values instead of just two as in the Ising model. The $q=2$ case is of course identical to the Ising case, apart from a scaling of the coupling constant by a factor of two. Also indicated in the plot are the exactly known critical temperatures. Nothing much changes qualitatively by increasing $q$, but the maxima become narrower and closer to the exact phase transition. It might be noted that for $q \leq 4$ the phase transition is a continuous phase transition, while for $q>4$ it is of first order, but this does not appear to reflect in any way in the behaviour of the mutual information.

A different generalization of Ising models is given by Potts models, governed by the Hamiltonian $H=$ $-J \sum_{\langle i j\rangle} \cos \left(s_{i}, s_{j}\right)$ where the $s_{k}$ are now angles $2 \pi k / q$. For higher $q$, this is in several ways a more complex model, which is why figure 5 first shows ours numerical results for the heat capacity. The behaviour of the heat capacity matches well that found in $23+25$. It indicates that the clock model does in fact have two phase transitions for $q>4$. However, these two critical points no longer coincide well with the maxima of the heat capacity; the dotted red lines show the locations of the phase transitions as estimated in 25]. For the $q=4$ case, the maximum of the heat capacity is a much better match for the location of the phase transition, which is known exactly in this case 26. Figure 6 then shows the corresponding mutual information plots. The $q=4$ case follows very much the usual pattern we have found before, with the phase transition occurring at the inflection point of the mutual information, but the meaning of the 


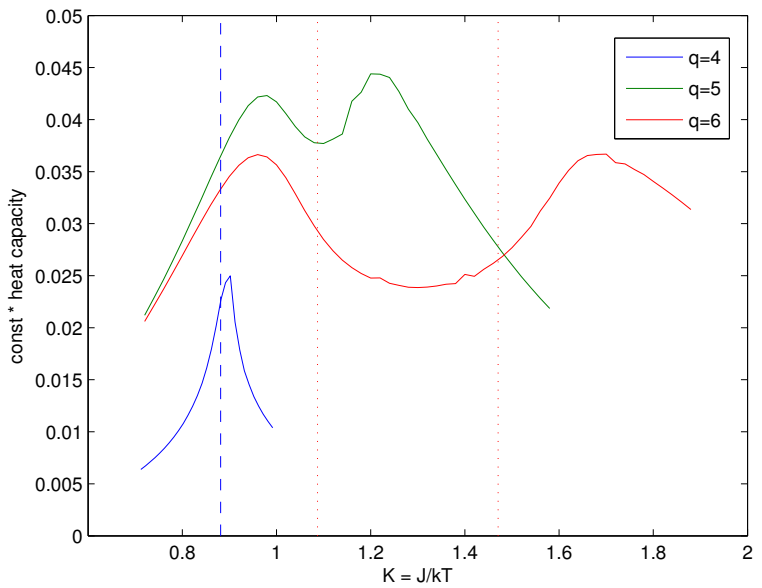

FIG. 5. Heat capacity for the clock model with $q=4,5,6$

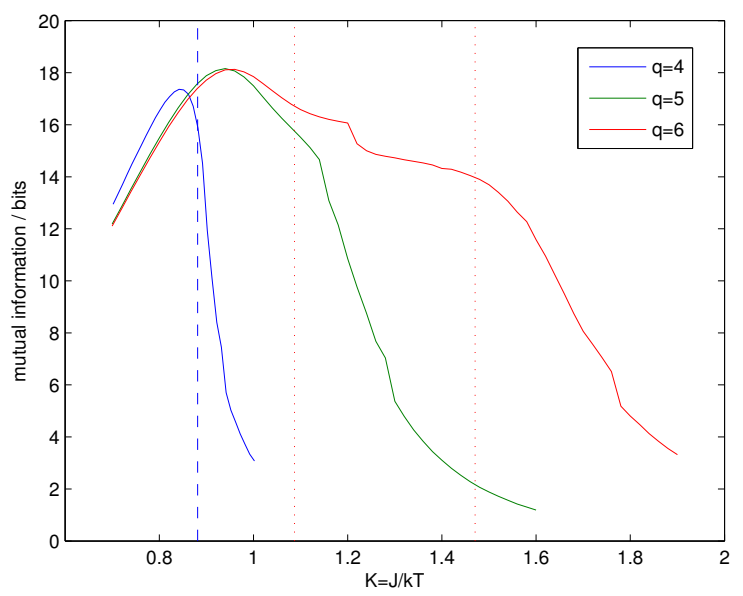

FIG. 6. Heat capacity for the clock model with $q=4,5,6$

curves for higher $q$ is essentially open for interpretation.

\section{RELATIONSHIP TO FORTUIN-KASTELEYN CLUSTERS}

Is there a way to understand why, in all the cases we have studied, the mutual information should have its maximum not at the phase transition, but in the hightemperature phase? We would like to suggest the idea to look at the Fortuin-Kasteleyn (FK) clusters [27] in the model. Those clusters are clusters of aligned spins, but not the simple geometric clusters that would arise from combining all neighbouring aligned spins. Instead, bonds between aligned spins only exist with a probability $1-\exp (\beta \Delta E)$ where $\Delta E$ is the energy difference between aligned and unaligned spins. Making bonds with this probability ensures that we only use "meaningful" bonds and not those existing just because two neighbour-

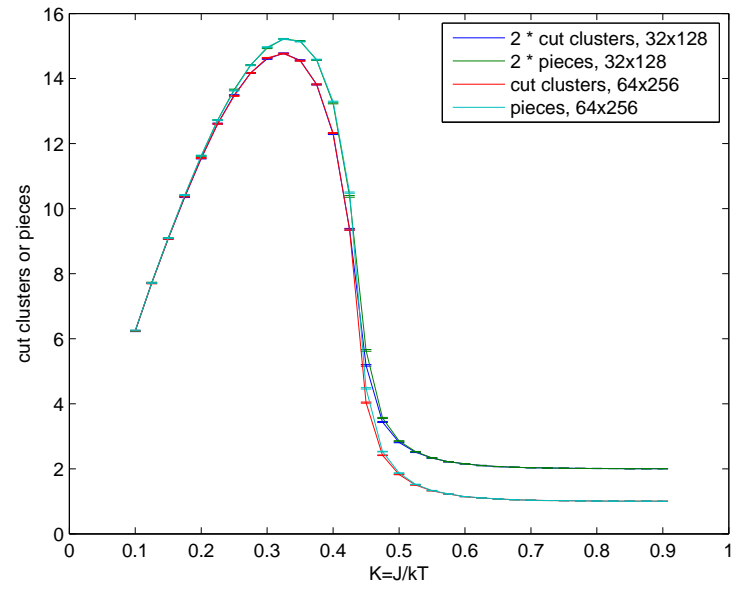

FIG. 7. FK clusters being cut (cylindrical geometry). The simulations were done using Swendsen-Wang [28] cluster updates, which also allow the immediate identification of clusters that are being cut. Code from the ALPS project [29, 30] was used in the simulations.

ing spins happen to be aligned. The resulting FK clusters can then be flipped independently from each other, which is the basis for efficient Monte Carlo cluster updates. In any given configuration during the Monte Carlo process, spins within a cluster are perfectly correlated, while spins in different clusters are uncorrelated.

While we cannot currently state an exact relation, it seems intuitive that the mutual information should be related to the number of such clusters that are cut when dividing the total system into its two subsystems. Possibly, an even better quantity to study would be the number of pieces that result from such a cut. The mutual information is certainly something more complicated than just the number of those pieces, but it might nevertheless help to give an insight of the factors at play: at very high temperature, FK clusters (unlike geometric clusters!) only consist of single spins, therefore no clusters will ever be cut, corresponding to no mutual information. At very low temperatures, there will just be one big cluster, corresponding to the limit of one bit (in Potts models: $q$-it) of mutual information. In between, there will be a maximum, where we have several clusters of nontrivial size that are being cut. Figure 7 shows this for the simple measures "number of clusters cut" or "number of pieces (on one side) after the cut", for the Ising model. While it is obvious that these measures do not show the same behaviour as the mutual information, it seems notable that they both also exhibit maxima that lie deeply in the paramagnetic phase, and there again appears to be a similar inflection point at the critical temperature. This could serve as a starting point for understanding this phenomenon. 


\section{CONCLUSION}

We have presented methods that allow to calculate mutual information in classical models. We examined the scaling behaviour in different phases and concluded that the system sizes we can study are a good approximation of the thermodynamic limit. We still would like to gain a better understanding of the maximum of the mutual information within the high-temperature phase. We are also still studying different models and anisotropic couplings, and are also working on extending our results to quantum rather than just classical models. For example, the 1+1-dimensional quantum Ising model is of course intimately related to the 2-dimensional classical Ising model studied in the present work, by a mapping that has also been exploited in 31 .

\section{ACKNOWLEDGMENTS}

We thank Hans Gerd Evertz, Maarten van den Nest, and Gerardo Ortiz for many valuable comments and discussions. This work is supported by the EU STReP QUE-VADIS, the ERC grant QUERG, the FWF SFB grants FoQuS and ViCoM, the FWF Doctoral Programme CoQuS (W 1210) and the Pauli Center at ETH Zurich.
[1] G. Vidal, J.I. Latorre, E. Rico, and A. Kitaev, "Entanglement in quantum critical phenomena," Physical Review Letters 90, 227902 (2003).

[2] P. Calabrese and J. Cardy, "Entanglement entropy and quantum field theory," Journal of Statistical Mechanics: Theory and Experiment 2004, P06002 (2004).

[3] M.B. Plenio, J. Eisert, J. Dreissig, and M. Cramer, "Entropy, entanglement, and area: analytical results for harmonic lattice systems," Physical Review Letters 94, 60503 (2005).

[4] A.R. Its, B.Q. Jin, and V.E. Korepin, "Entanglement in the XY spin chain," Journal of Physics A: Mathematical and General 38, 2975 (2005).

[5] A. Kitaev and J. Preskill, "Topological entanglement entropy," Physical Review Letters 96, 110404 (2006).

[6] M. Levin and X.G. Wen, "Detecting topological order in a ground state wave function," Physical Review Letters 96, 110405 (2006).

[7] F. Verstraete and J.I. Cirac, "Matrix product states represent ground states faithfully," Physical Review B 73, 94423 (2006).

[8] F. Verstraete, M.M. Wolf, D. Perez-Garcia, and J.I. Cirac, "Criticality, the area law, and the computational power of projected entangled pair states," Physical Review Letters 96, 220601 (2006).

[9] M.B. Hastings, "An area law for one-dimensional quantum systems," Journal of Statistical Mechanics: Theory and Experiment 2007, P08024 (2007).

[10] R.G. Melko, A.B. Kallin, and M.B. Hastings, "Finitesize scaling of mutual information in Monte Carlo simulations: Application to the spin-1/2 XXZ model," Physical Review B 82, 100409 (2010).

[11] M.B. Hastings, I. González, A.B. Kallin, and R.G. Melko, "Measuring Renyi Entanglement Entropy in Quantum Monte Carlo Simulations," Physical Review Letters 104, 157201 (2010).

[12] J. Eisert, M. Cramer, and M.B. Plenio, "Colloquium: Area laws for the entanglement entropy," Reviews of Modern Physics 82, 277-306 (2010).

[13] M.M. Wolf, F. Verstraete, M.B. Hastings, and J.I. Cirac, "Area laws in quantum systems: mutual information and correlations," Physical Review Letters 100, 70502 (2008).

[14] R. König, R. Renner, and C. Schaffner, "The operational meaning of min-and max-entropy," IEEE Transactions on Information Theory 55, 4337-4347 (2009).

[15] T. Nishino, "Density matrix renormalization group method for 2D classical models," Journal of the Physical Society of Japan 64, 3598-3601 (1995).

[16] T. Nishino and K. Okunishi, "Product wave function renormalization group," Journal of the Physical Society of Japan 64, 4084-4087 (1995).

[17] F. Verstraete, V. Murg, and J.I. Cirac, "Matrix product states, projected entangled pair states, and variational renormalization group methods for quantum spin systems," Advances in Physics 57, 143-224 (2008).

[18] B. Pirvu, F. Verstraete, and G. Vidal, "Exploiting translational invariance in Matrix Product State simulations of spin chains with periodic boundary conditions. arXiv:1005.5195,".

[19] M.E. Fisher, "On the dimer solution of planar Ising models," Journal of Mathematical Physics 7, 1776 (1966).

[20] B.M. McCoy and T.T. Wu, The two-dimensional Ising model (Harvard University Press, 1973).

[21] J.I. Latorre, R. Orús, E. Rico, and J. Vidal, "Entanglement entropy in the Lipkin-Meshkov-Glick model," Physical Review A 71, 64101 (2005).

[22] J. Vidal, S. Dusuel, and T. Barthel, "Entanglement entropy in collective models," Journal of Statistical Mechanics: Theory and Experiment 2007, P01015 (2007).

[23] J. Tobochnik, "Properties of the q-state clock model for q=4, 5, and 6," Physical Review B 26, 6201-6207 (1982).

[24] J. Tobochnik, "Erratum: Properties of the q-state clock model for q=4, 5, and 6," Physical Review B 27, 6972 (1983).

[25] M.S.S. Challa and D.P. Landau, "Critical behavior of the six-state clock model in two dimensions," Physical Review B 33, 437-443 (1986).

[26] M. Suzuki, "Solution of Potts Model for Phase Transition," Progress of Theoretical Physics 37, 770-772 (1967).

[27] C.M. Fortuin and P.W. Kasteleyn, "On the randomcluster model:: I. Introduction and relation to other models," Physica 57, 536-564 (1972).

[28] R.H. Swendsen and J.S. Wang, "Nonuniversal critical dynamics in Monte Carlo simulations," Physical Review Letters 58, 86-88 (1987).

[29] F. Albuquerque et al. (ALPS collaboration), "The ALPS project release 1.3: open source software for strongly cor- 
related systems," Journal of Magnetism and Magnetic Materials 310, 1187 (2007).

[30] M. Troyer, B. Ammon, and E. Heeb, "Parallel object oriented Monte Carlo Simulations," Lecture Notes in Com- puter Science 1505, 191 (1998).

[31] J.M. Stéphan, S. Furukawa, G. Misguich, and V. Pasquier, "Shannon and entanglement entropies of one-and two-dimensional critical wave functions," Physical Review B 80, 184421 (2009). 